\title{
Descartes' Contributions to Optics
}

\author{
BY DR. J. F. SCOTT
}

\section{ABSTRACT of Paper read on 23rd October, 1950}

Descartes' contributions to Optics, though not of equal weight as his contributions to mathematics, are nevertheless of great importance. Their value lies not so much in any marks of originality as in the impulse which they gave to the practical aspect of the subject.

The general principles of reflexion and refraction appear to have been well understood long before Descartes directed his attention to the subject. The equality of the angles of incidence and reflexion was known in antiquity, and the apparent bending of a stick in water, and the disappearing coin illusion were accepted as evidence that the ray was bent on passing from one medium to another. But all attempts at discovering a law of refraction had proved abortive ; moreover, no one before Descartes appears to have made any attempt to assign a cause to the bending of the ray as it passed from one medium to another. Despite his conviction that light is a " pressure " emanating from the luminous body, Descartes was never able to shake himself free from an emission theory and in his explanation of reflexion and refraction he compares the ray of light to the motion of a ball striking a surface obliquely. If the ball is reflected, it is easy to show that the angles of incidence and reflexion will be equal. But if it passes through the surface its speed will be altered, and with its speed, its direction. Proceeding on these lines Descartes arrived at the conclusion that " when rays pass obliquely from one medium to another which offers greater or less resistance, they are turned in such a way that they are always less inclined on the side where they pass more easily and in proportion as they pass more easily. But it is to. be noted that this inclination is to be measured by the magnitude of the right lines CB and $\mathrm{EF}^{*}$, or lines similar to these, and not by the angles since the ratio of these angles is not constant. Moreover for the same two media the ratio of these two right lines is constant for all angles of incidence, and its value may be deduced by trial ". This is the first appearance of the law of refraction, the discovery of which, however, is usually attributed to Snell. Snell was Professor of Mathematics at Leyden from 1615 till his death in 1626 and it was probably during his professorship that he hit upon the law of refraction. His work, however, was not published; nevertheless it is difficult to believe that his contemporaries long remained in ignorance of it, particularly as, if Vossius (De Lucis Natura et Proprietate. Amsterdam 1662) is right, it was publicly taught throughout Holland by Hortensius, a pupil of Snell and an editor of some of his works. Christian Huygens, whose father Constantyn Huygens was singularly well placed among the learned of the day, shared the view that Descartes was familiar with the results of Snell's researches. (See D.J. Kort:weg: Descartes et les Manuscrits de Snellius. Revue de Métaphysique et de Morale, 1896 : also, Huygens : Oeuvres, Vol. 13, Fasc. 1, p. 19).

On the other hand, it is clear from his correspondence that Descartes was in possession of the law as early as 1629 , and all the evidence points to the fact that he did not hear of Snell's discovery until 1632, by which time his own researches were probably so far advanced that he had little to learn from Snell. This would clear Descartes of any charge of plagiarism, and it is significant to note that no one during his lifetime appears to have suggested that he had appropriated Snell's results. Leaving aside the question of priority there is no question as to the superiority of Descartes' investigation over that

* Compare the usual geometrical construction for measuring the refractive index; 
of Snell. Snell drew no conclusion from his discovery, nor did he connect it with any principle. Descartes' investigations on the other hand were the starting point of a series of deductions which have proved extremely fruitful. A notable example is his explanation of the formation of the rainbow, where not only did he handle the law with skill, but also made use of a very accurate value of the refractive index of water (Les Météores, Disc. viii). His assumption, too, that the bending of the ray was a consequence of a change in the speed of transmission, and further, that this speed of transmission depended only upon the nature of the medium it traversed, marked a notable advance. Nevertheless, he does not appear to have been particularly happy in his views upon the speed of light. It is generally believed that he regarded the speed of transmission as infinite, this upon the strength of an observation that " the light can extend its rays in an instant from the sun right up to our eyes" as well as a clear statement to the same effect in Ch. xiii of Le Monde, where the properties of light are enumerated. But his correspondence clearly shows that by an instant he meant an incredibly short space of time, so short in fact that it was useless to try to detect, still less measure, the duration of its passage from one terrestrial object to another.

A more serious blemish lay in his persistance in making the light travel faster in the dense medium than in the rare one. Light, he maintained, is an action upon the subtle matter which fills the pores of bodies, and since the pores of a body like air are soft and ill-joined, the subtle matter between its particles is less disposed to respond to this action than is the case with a harder and firmer body ; consequently in the case of the latter the more readily does the light pass through, just as a ball rolls more easily on a hard floor than on a carpet.

The "explanation ", however, did not satisfy his contemporaries, particularly Fermat. Fermat argued that the principle which came ultimately to be known as the Principle of Least Action, and which was long known to operate in the case of reflexion, would still hold if the light passed from one medium to another, so that light travelling from a point in one medium to a point in another would so adjust its path that it would traverse the distance in the shortest possible time. Under these circumstances, Fermat showed that the resistances encountered in the two media would be inversely proportional to the angles of incidence and refraction. Descartes wrongly believed the speeds to be inversely proportional to these same angles and he further maintained that light must travel more readily through water than through air and still more readily through glass, conclusions which were experimentally disproved by Foucault in $\mathbf{1 8 5 0}$.

Descartes' efforts to design lenses which would improve the telescope and the microseope, though ingenious, yielded no results of any practical value. The grinding of non-spherical lenses is still impracticable ; moreover, even if the technical difficulties could be overcome, the shapes suggested by Descartes did not diminish, in fact they rather increased, the defect known as chromatic aberration. Nevertheless his views, based as they were on sound mathematical and physical principles, helped to prepare the way for the optical discoveries of Hooke, Grimaldi and Newton. 